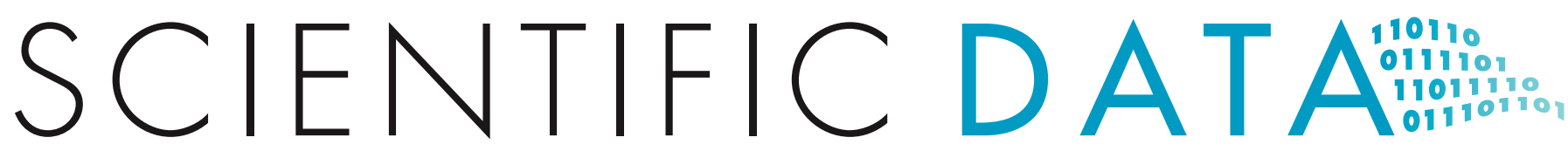

OPEN Data Descriptor: Image-based síRNA screen to identify kinases regulating Weibel-Palade body size control using electroporation

Received: 27 July 2016 Accepted: 22 December 2016

Published: 1 March 2017 Updated: 27 June 2017

\section{Robin Ketteler ${ }^{1}$, Jamie Freeman ${ }^{1}$, Nicola Stevenson ${ }^{1}$, Francesco Ferraro ${ }^{1}$, Nicole Bata ${ }^{1}$, Dan F. Cutler ${ }^{1}$ \& Janos Kriston-Vizi ${ }^{1}$}

High-content screening of kinase inhibitors is important in order to identify biogenesis and function mechanisms of subcellular organelles. Here, we present a human kinome siRNA high-content screen on primary human umbilical vein endothelial cells, that were transfected by electroporation. The data descriptor contains a confocal fluorescence, microscopic image dataset. We also describe an open source, automated image analysis workflow that can be reused to perform high-content analysis of other organelles. This dataset is suitable for analysis of morphological parameters that are linked to human umbilical vein endothelial cell (HUVEC) biology.

\begin{tabular}{|l|l|}
\hline Design Type & screening campaign • RNAi screening \\
\hline Measurement Type(s) & subcellular morphology \\
\hline Fechnology Type(s) & spinning-disk confocal microscope \\
\hline Sample Characteristic(s) & gene_silenced_by_RNA_interference \\
\hline
\end{tabular}




\section{Background \& Summary}

Microscopy-based high-content screening has emerged as a powerful method to interrogate biological pathways and to identify novel genes involved in cellular processes ${ }^{1}$. A common method that has found wide spread application is using short interfering RNA libraries ${ }^{2,3}$, and more recently, the use of CRISPRbased arrayed libraries has been suggested ${ }^{4}$. One caveat in the use of such methods is the high degree of variability between samples, often due to inherent biological variation or technical limitations. Several computational methods have aimed to reduce such variability, which can be applied post-screening to extracted datasets and improve analysis without the need to re-acquire images ${ }^{5,6}$. Of interest, many highcontent datasets only report few parameters and defeat the concept of multiparametric analysis that is possible with acquired images ${ }^{7}$. Thus, it can be useful to publish images from high-throughput screens in order to enable re-analysis and generation of novel insights by de novo analysis using alternative parameters. Furthermore, the analysis of a large number of objects in an image dataset sometimes enables unexpected discoveries. For instance, we have previously assessed the size of Weibel-Palade bodies (WPB) and identified a periodic length distribution when a large number of objects were analysed ${ }^{8}$. WeibelPalade bodies are secretory storage granules in endothelial cells that have an important role in primary haemostasis. WPBs are the endothelial storage organelles of von-Willebrand factor (vWF), a large adhesive glycoprotein that is released upon vascular injury and promotes the recruitment of platelets to the site of injury, thereby initiating primary haemostasis. WPBs can be visualised in cells by vWF immuno-staining and therefore factors that regulate this WPB formation can be identified by high-content screening approaches. We have previously monitored the length of WPBs in cells by high-throughput morphometry and identified a role for the Golgi ribbon in determining the size of these structures. This has led to novel insights into the biogenesis and function of these organelles with the concept of vWF cargo 'quanta' that form at the Golgi and can be packaged together during biogenesis WPBs, generating organelles of varying sizes ${ }^{8}$. Following this work, we have explored the possibility that WPB size may be regulated by kinases and performed a kinome siRNA screen in primary Human Umbilical Vein Endothelial Cells (HUVECs). Other works have identified GRK2 (ref. 9) and PI4-kinase ${ }^{10}$ kinases as regulators of WPB biogenesis and vWF release. We used electroporation as a technique to improve transfection efficiency into primary cells ${ }^{11}$ and applied an image analysis pipeline that is suitable for the analysis of intracellular organelle size and number.

We recorded a dataset of 10,560 images in four microscope channels monitoring WPBs, nuclei, trans-Golgi network and plasma membrane. We developed an image analysis pipeline to determine the size and length of Weibel-Palade Bodies. Positive (siRNA knockdown of vWF, Fig. 1a) and negative (control siRNA, Fig. 1b) controls were used to determine the suitability of this assay for monitoring changes in a large-scale screening format. Overall, more than 10 million objects were identified as WPBs and measured for intensity, Feret's diameter, and other parameters, listed in Table 1. In addition, we also monitored key cellular features such as cell number to account for effects on cell viability, a common phenotype observed in siRNA knockdown screens. We confirmed GRK2 as a positive hit and identified other potential candidate kinases that regulate WPB length and number (Table 2). When assessing the reproducibility among biological replicates, we observed a high level of variability between replicates that might reflect technical limitations of the electroporation. Another possibility is that the low dynamic range observed in this assay reflects inherent features of the underlying biology, e.g. that multiple kinases may only have a minor impact on WPB formation. Nonetheless, this dataset can be used to extract other morphological features that are not directly linked to WPB biology. Alternatively, post-screening methods that enable the separation of on- and off-target effects may greatly benefit the analysis. We therefore make this dataset publicly available to encourage re-analysis by means of alternative methods.
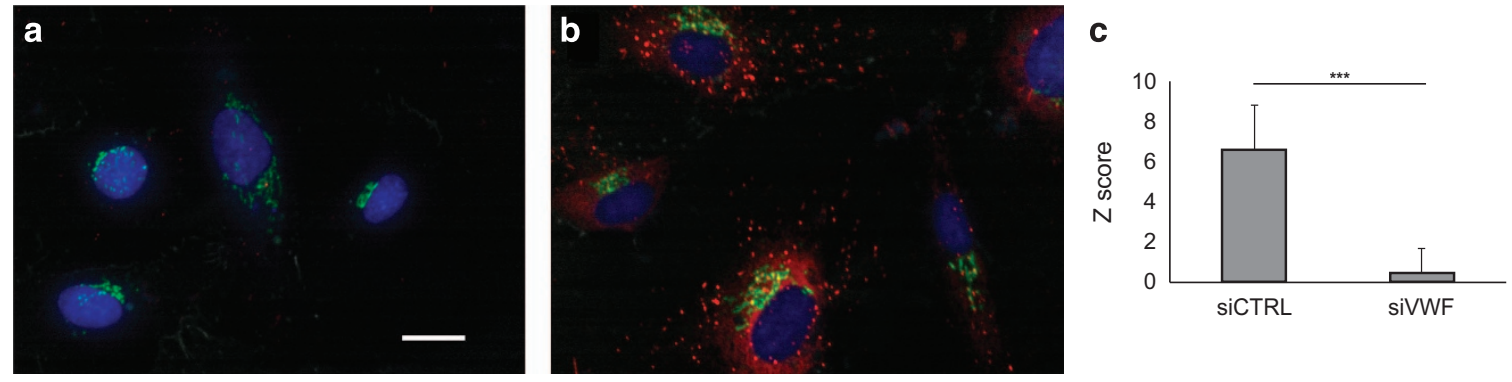

Figure 1. Representative WPB phenotypes and quantification of positive and negative controls. (a) siRNA (negative control) (b) siRNA knockdown of vWF (positive control). Nuclei (blue), trans-Golgi network (green), WPB (red) and plasma membrane (grey) were stained in HUVEC cells. Scale bar $20 \mu \mathrm{m}$. The figures show representative, cropped regions of fields of view, that allowed the proper visualization of the small WPBs.

(c) The intensity Z scores (3_TotalWPBrawIntDenPerCellNR) of negative control wells (siCTRL) are significantly higher than that of positive control wells (siVWF). Mean \pm S.D. plotted for 88 independent biological replicates for each condition, two-tailed Student's two sample t-test $P<2.2 \mathrm{e}-16$. 


\begin{tabular}{|c|c|}
\hline label & description \\
\hline Area & Area of a WPB object in $\mu \mathrm{m}^{2}$. \\
\hline Mean & Average grey intensity of a WPB object [relative fluorescence unit r.f.u.] \\
\hline StdDev & Standard deviation of a WPB object pixel intensity [r.f.u.]. \\
\hline Min & Minimum intensity of a WPB object [r.f.u.]. \\
\hline Max & Maximum intensity of a WPB object [r.f.u.]. \\
\hline $\mathrm{X}$ & The X coordinate of a WPB object's centroid [pixel]. \\
\hline Y & The Y coordinate of a WPB object's centroid [pixel]. \\
\hline Perim. & Perimeter of a WPB object $\left[\mu \mathrm{m}^{2}\right]$. \\
\hline Major & Length of primary axis of the best fitting ellipse around a WPB object $[\mu \mathrm{m}]$. \\
\hline Minor & Length of secondary axis of the best fitting ellipse around a WPB object $[\mu \mathrm{m}]$. \\
\hline Angle & $\begin{array}{l}\text { Angle between the horizontal axis of the image and the primary axis of the best fitting ellipse around a WPB } \\
\text { object [degree]. }\end{array}$ \\
\hline Circ. & Circularity shape descriptor of a WPB. Circ. $=1$ means a perfect circle. \\
\hline Feret & The longest diameter between any two points along a WPB's boundary $[\mu \mathrm{m}]$. \\
\hline IntDen & The product of a WPB's area and mean grey intensity [r.f.u.]. \\
\hline Median & Median grey intensity of a WPB object [r.f.u.]. \\
\hline RawIntDen & The sum of the values of the pixels in a WPB [r.f.u.]. \\
\hline Slice & The number of image in an image stack. \\
\hline FeretX & The X coordinate of the end of a WPB's Feret diameter [pixel]. \\
\hline FeretY & The Y coordinate of the end of a WPB's Feret diameter [pixel]. \\
\hline FeretAngle & Angle between the horizontal axis of the image and the Feret's diameter of a WPB object [degree]. \\
\hline MinFeret & The shortest diameter between any two points along a WPB's boundary $[\mu \mathrm{m}]$. \\
\hline AR & $\begin{array}{l}\text { Aspect ratio is a shape descriptor of a WPB, means the ratio of the length of the primary and secondary } \\
\text { axes of a WPB's fitted ellipse. }\end{array}$ \\
\hline Round & Roundness is a shape descriptor of a WPB, the inverse of AR. \\
\hline Solidity & The ratio between a WPB's area and convex area. \\
\hline
\end{tabular}

Table 1. WPB features quantified by ImageJ The features listed as headings and are described at the ImageJ documentation website: https://imagej.nih.gov/ij/docs/guide/146-30.html\#toc-Subsection-30.7 (accessed on 09/02/2017).

In summary, we present here data from a siRNA screen targeting the human kinome in order to identify regulators of Weibel-Palade body formation. The screen was performed in primary human umbilical vein endothelial cells (HUVECs), a cell type that has been difficult to transfect with common lipofection methods ${ }^{12}$. We therefore used electroporation with the 96-well nucleofector machine and achieved good knockdown efficiency using the positive control (vWF-targeting siRNA) (Fig. 1). We developed an image analysis pipeline in ImageJ and identified several kinases that regulate the area, number, length and intensity of WPB objects. We therefore propose that the formation of WPBs is regulated by kinases. Further, we demonstrate that electroporation can be used in a high-content screening workflow to generate data from difficult to transfect cell types. And finally, we present an image analysis workflow for the identification and analysis of key features of cellular organelles, in this case WPBs, that can be applied to other sub-cellular structures in high- or low-throughput settings.

\section{Methods}

\section{Electroporation and Screening}

Primary HUVECs were transfected in suspension using the Lonza/Amaxa nucleofector 96-well shuttle. The human kinome MISSION siRNA kinase panel library from Sigma-Aldrich was used in this study (Supplementary Table 1). Three oligonucleotide pairs were provided for each gene in a total of 33 plates. A pool of three siRNA molecules targeting the same gene was generated by mixing equal amounts of the three individual siRNA oligonucleotides together, generating 11 'pool' plates that were used for screening. Control siRNAs were custom synthesised, as described ${ }^{8}$. An siRNA targeting firefly luciferase was used as a negative control and siRNA targeting vWF as positive control. Cells were thawed and cultured for one week prior to the screen, low passage, P3-P5 cells were used. Briefly, 75,000 cells were mixed with $20 \mu \mathrm{l}$ nucleofection buffer and the pre-defined HUVEC nucleofection protocol on the instrument was used for electroporation of $30 \mathrm{pmol}$ siRNA, in agreement with standard nucleofection protocols used in other screens ${ }^{12}$. Cells were seeded into 96 well, optically clear bottom, tissue culture treated, sterile, polystyrene (Nunc, product number 167008) microplates that were pre-coated with gelatin and allowed to adhere 


\begin{tabular}{|c|c|c|c|}
\hline $\mathrm{Z}$ score & plate & well & GeneSymbol \\
\hline 6.88 & 6 & A07 & DYRK4 \\
\hline 5.33 & 11 & E05 & LMTK3 \\
\hline 5.01 & 6 & B05 & CDK10 \\
\hline 3.52 & 11 & E02 & SBK1 \\
\hline 3.47 & 11 & $\mathrm{C} 04$ & PRKG1 \\
\hline 3.31 & 6 & F02 & PIP5K1B \\
\hline 3.16 & 6 & $\mathrm{C} 05$ & CDK10 \\
\hline 3.12 & 11 & $\mathrm{~F} 03$ & PIP5K1A \\
\hline 3.03 & 6 & B02 & BRD3 \\
\hline 2.95 & 11 & B04 & RAPGEF3 \\
\hline 2.92 & 11 & $\mathrm{E} 07$ & CSNK2A1 \\
\hline 2.85 & 6 & F11 & MAP4K4 \\
\hline 2.85 & 6 & B08 & HERC2 2 \\
\hline 2.76 & 8 & A08 & TRIM33 \\
\hline 2.69 & 7 & F04 & HIPK3 \\
\hline 2.64 & 8 & $\mathrm{~A} 10$ & TRPM7 \\
\hline 2.57 & 1 & A03 & ADRBK1 \\
\hline 2.57 & 6 & F03 & MAP4K3 \\
\hline 2.56 & 8 & $\mathrm{C} 09$ & PANK1 \\
\hline 2.51 & 9 & F04 & ADCK1 \\
\hline 2.5 & 8 & $\mathrm{~A} 02$ & SGK3 \\
\hline 2.47 & 6 & $\mathrm{C} 06$ & $\mathrm{CDC} 2 \mathrm{~L} 5$ \\
\hline 2.34 & 11 & F06 & DYRK1B \\
\hline 2.28 & 6 & G05 & CASK \\
\hline 2.24 & 3 & B09 & NEK2 \\
\hline 2.24 & 3 & F11 & PCTK2 \\
\hline 2.23 & 6 & G07 & STK19 \\
\hline 2.22 & 1 & A04 & AMHR2 \\
\hline 2.21 & 10 & $\mathrm{C} 06$ & NEK7 \\
\hline 2.17 & 8 & B05 & TBK1 \\
\hline 2.16 & 9 & E07 & WNK2 \\
\hline 2.16 & 10 & $\mathrm{C} 09$ & PRPS1L1 \\
\hline 2.16 & 9 & E08 & SGK269 \\
\hline 2.16 & 6 & G08 & CDKL2 \\
\hline 2.08 & 2 & A06 & MARK2 \\
\hline 2.06 & 11 & D04 & PRKDC \\
\hline 2.04 & 2 & B05 & DMPK \\
\hline 2.02 & 7 & H05 & RAPGEF3 \\
\hline 2.02 & 6 & A06 & STK16 \\
\hline 2.01 & 10 & B04 & EVI5L \\
\hline
\end{tabular}

Table 2. GRK2 is identified as a hit regulating WPB number Hitlist show positive hits, that were thresholded as Z score $>2$. GRK2 (syn. ADRBK1) was confirmed as one of the top hits.

overnight. Media was replaced the following day, and cells were fixed in 4\% PFA and stained $48 \mathrm{~h}$ after electroporation. The $48 \mathrm{~h}$ time frame is well in line with recommendations for siRNA screening (e.g. Martin et al. ${ }^{13}$, which recommends $48-120 \mathrm{~h}$ for screening). Immunostaining was performed as described $^{8}$. Briefly, we used Hoechst33342 for nuclear staining, rabbit anti-vWF antibody for WPBs, sheep anti-TGN-46 antibody for the trans-Golgi network, and mouse anti-ve-Cadherin antibody for plasma membrane staining. The following antibodies were used: anti-vWF, rabbit polyclonal (Dako, cat. no. A0082), anti-TGN46, sheep polyclonal antibody (AbD Serotec, cat. no. AHP500) and anti-VE-cadherin, mouse monoclonal antibody (BD Biosciences, cat. no. 555661). 


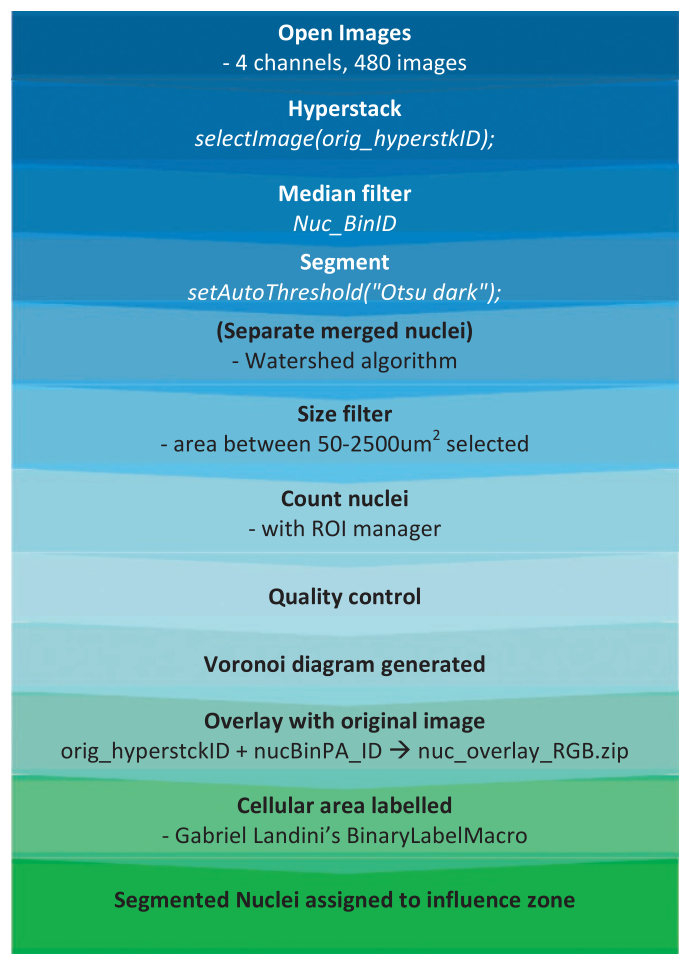

Figure 2. Diagram depicting the image processing workflow by ImageJ.

\section{High-content image acquisition}

Images were acquired with a spinning disk confocal Opera LX plate reader microscope (PerkinElmer) with Olympus 40x LUCPLFLN (NA=0.6) air lens at four fluorescence channels using $365 \mathrm{~nm}, 488 \mathrm{~nm}$, $561 \mathrm{~nm}$ and $640 \mathrm{~nm}$ excitation wavelengths respectively. Camera binning was not used, resulting in a nominal pixel size of $0.1615 \mu \mathrm{m}$. We worked with confluent cells, $70-100$ cells were imaged in the 5 fields of view of the wells.

The manufacturer's recommended procedures (described in Opera Quick Guide 1.8.1, product number HH10940200) and Opera Adjustment Plate (PerkinElmer, product number HH10000650) was used for optical corrections. In order to compensate the systematic uneven brightness distribution of the optics, reference images were taken for flatfield correction. The 'fish eye effect' and offset between channels was compensated using bead images for skewcrop analysis. Both flatfield correction and skewcrop analysis was done before image acquisition of each experiment. Five field of views (FoVs) were acquired in each well.

The microscopy exposure times and laser powers were used as follows. $250 \mathrm{~ms}$ exposure time was used at the $365 \mathrm{~nm}$ channel for nuclear imaging, $2000 \mathrm{~ms}$ with $3620 \mu \mathrm{W}$ laser power at the $488 \mathrm{~nm}$ channel for trans-Golgi network (TGN46), $2000 \mathrm{~ms}$ with $1640 \mu \mathrm{W}$ laser power at the $561 \mathrm{~nm}$ channel for WPB (vWF staining) and $2000 \mathrm{~ms}$ with $862 \mu \mathrm{W}$ laser power at the $640 \mathrm{~nm}$ channel for plasma membrane (VE-cadherin). The screen was done in two batches (pools A-F on one day and G-K on another day).

The 4-channel, fluorescence, 12 bit depth images were acquired as Opera ${ }^{*}$.flex files and analysed after conversion into ${ }^{\star}$.tif format by the Acapella FlexToVolocity.script.

\section{High-content analysis}

Image processing. Hardware specifications of the high-content-analysis computer: A highperformance computer was built on a Z8PE-D18 server motherboard (Asus) equipped with 145 GB memory, two Xeon E5520 CPUs (Intel) running at $2.27 \mathrm{GHz}$ clock rate.

ImageJ macro. The macro (Fig. 2) uses 45 GB memory, for automatically analysing the 480 images of a whole 96 well plate, in $30 \mathrm{~min}$. For the Bernsen thresholding, it requires Gabriel Landini's Auto Local Threshold plugin (http://imagej.net/Auto_Local_Threshold) to be installed to ImageJ. Alternatively, the Auto Local Threshold plugin is integrated into Fiji (http://fiji.sc). The macro requires subfolders in the results folder named as: influzone, overlay_nuc_RGB, overlay_vWF_RGB, Results_Features, Results_Nuc_Features, Results_WPB_cell_ID and RoiSet.

The macro has two main parts. The first part segments the nuclear channel and approximates individual cellular area using the midlines between nuclei. The second part analyses the WPB channel. 
a

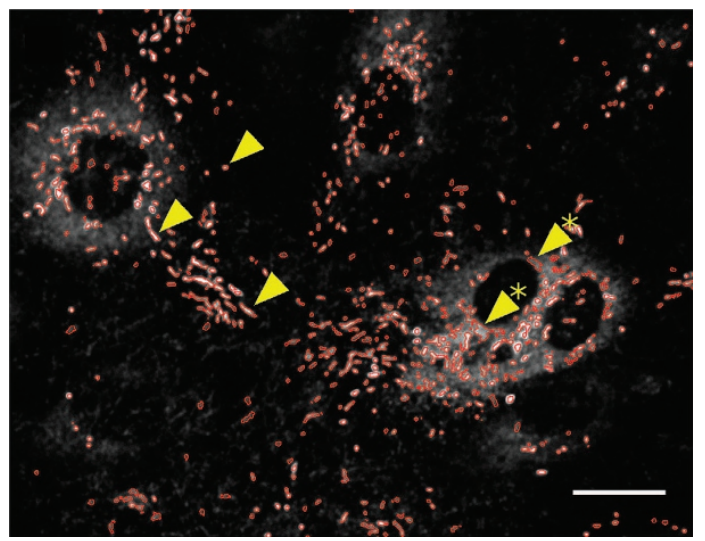

C

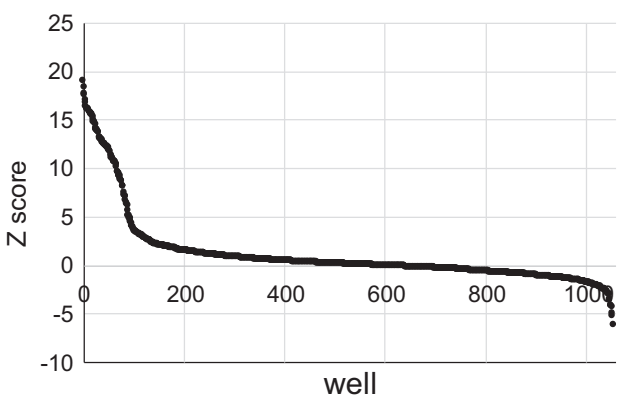

3_TotalWPBrawIntDenPerCellNR

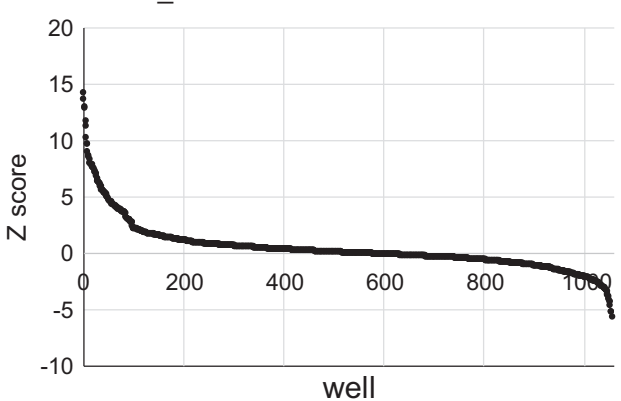

b

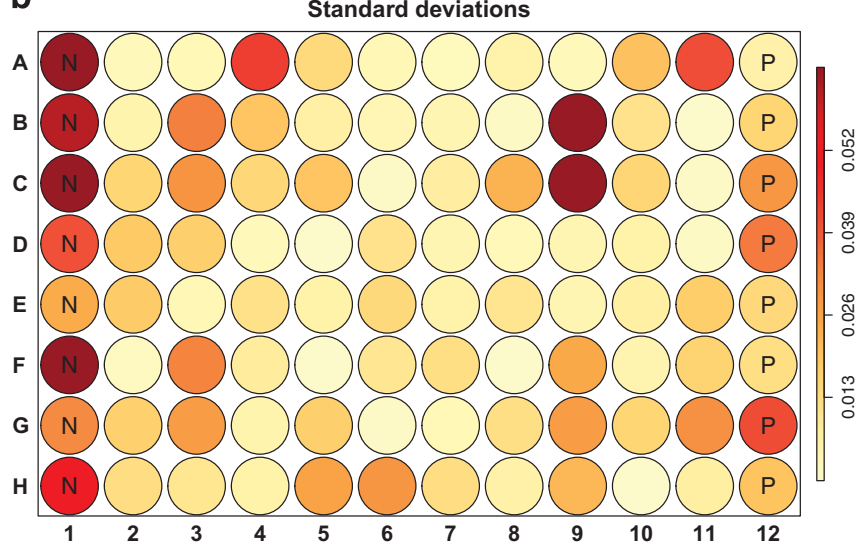

2_WPBnrPerCellNR

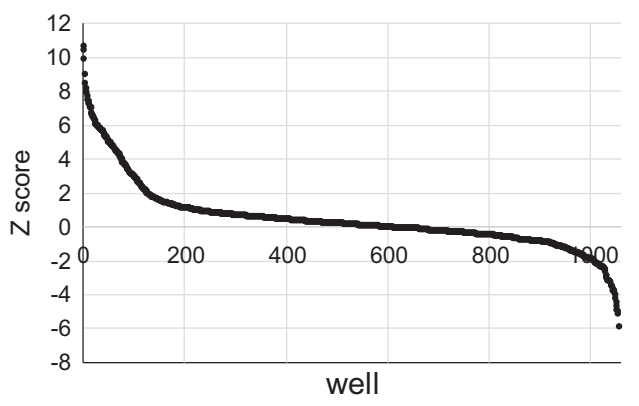

4_TotalFoVpercentWBPnr

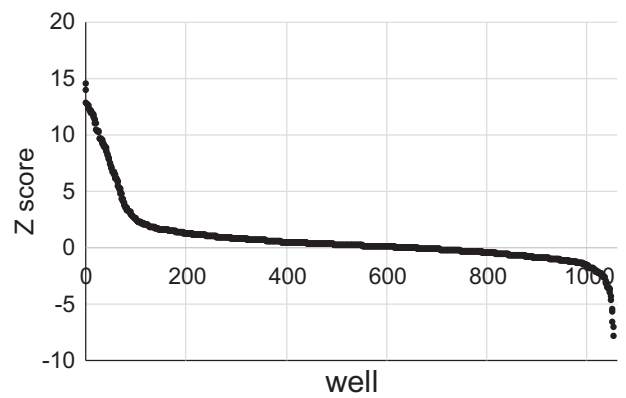

Figure 3. Electroporation-based siRNA screen result. (a) Segmentation quality control overlay image. The red contour indicates the boundary of segmented WPBs. Representative image, the overlay of all processed images are available in the repository as *_vWF_overlay_RGB.zip files and the WPB contours as *_RoiSet.zip files (Data Citation 1). Arrows denote correctly segmented representative WPBs, arrows with star denote nonWPB vWF objects as segmentation artefacts. Scale bar $20 \mu \mathrm{m}$. (b) Reproducibility is quantified by calculating the standard deviation of per well values across the replicates. A representative plate heatmap is presented here and the data for all plates are available in the repository as components of the HTML reports. Negative control wells are labelled with 'N' and 'P' stands for positive control wells. (c) Distribution of the 1056 well's Z scores in the 1_PercentWPBarea dataset. All Z score values are available in the HTML reports (Data Citation 1).

The nuclear channel was pre-processed using a Median filter with 2 pixel radius in order to reduce the inherent noise in the image and smoothen the nuclear contours. The latter is a necessary step because of the separation mechanism of touching nuclei. Watershed algorithm was used to separate merged nuclei based on convexity and smoothened contours eliminate separating artefacts caused by minor concavities on a coarse nuclear contour. A size filter eliminates artefact objects that cannot be considered as nuclei, being too small $\left(<50 \mu \mathrm{m}^{2}\right)$ or too large $\left(<2500 \mu \mathrm{m}^{2}\right)$.

Quality control (QC) plays an important role in automated image processing. Contour overlays on the original images serve as a convenient, quick visual QC method and helps to find the optimal parameters at the image processing step. 
As a QC of the nuclear segmentation, the contours of the nuclei overlaid on the original nuclear images were automatically saved by the macro.

Delaunay Triangulation was used to generate a Voronoi diagram i.e. the midlines between nuclei centroids in order to generate influence zones of nuclei that approximate individual cell contours. There are advantages of this approach: it is simple, allows single cell analysis, requires nuclear stain only, and is computationally cheap. It is limited though: it follows the real cell boundaries only if those lay on the midline between the nuclei and it assumes that the cells form a confluent monolayer. Practically, the latter shortcoming is significant only if the precise quantification of cellular area is needed. In this screen this feature was only used as a proof of concept to provide a simple initial approach for the development of a more precise plasma membrane delineating pipeline. The Voronoi mesh was overlaid on the original nuclear channel for each image and the result was saved as an image stack (see the *_nuc overlay_RGB.zip files (Data Citation 1)).

In order to assign its cell identifier to each WPB pixel, the cellular area was labelled using code from Gabriel Landini's BinaryLabelMacro (http://sites.imagej.net/Landini/plugins/Morphology/BinaryLabelMacro.ijm-20140627120652). Our image processing workflow uses grey shades to label cells. An 8 bit image allows the use of $2^{8}=256$ grey shades, that can be a rate limiting factor when the image has more than 256 cells. Therefore, we use 16 bit images, that allow the labeling of more than 256, maximum $2^{16}=65535$ cells in an image. The labels were encoded into 16 bit range values, with 0 for the separating Voronoi mesh. The 16 bit depth labelling was used for computational purposes, automated contrast stretch was applied when the labelled images were visualised and saved in RGB stack format (see the ${ }^{*}$ influzone.zip files (Data Citation 1)).

Furthermore, the segmented nuclei were assigned to their corresponding influence zone by measuring the mean intensity on influence zone image. The results were saved in the *_Results_Nuc_Features.csv files (Data Citation 1).

At this point, the image processing workflow was ready for the WPB channel quantification. A copy was created about the second (WPB) channel in order to preserve the original pixel intensities so intensity features can be measures after the segmentation. Subsequently, the thresholding was performed on the duplicated stack. Noise reduction was performed using the rolling ball algorithm ${ }^{14}$ with 1 pixel as rolling ball radius parameter and using sliding paraboloid correction. We used a Bernsen local thresholding ${ }^{15}$ with the default contrast threshold parameter value (15) on the 8 bit converted WPB images because we found that it provided us with an acceptable precision. The contrast threshold is the only parameter value that might need adjustment in the entire image processing macro. If the fluorescence signal is too weak, then the Bernsen algorithm becomes 'too sensitive' and detects even the out of focus and background pixels. In that case the user needs to set the algorithm 'insensitive' by increasing the contrast threshold parameter from the default 15 value to 30 or 50. The WPB pixels overlapping the Voronoi mesh were deleted in order to avoid the ambiguous situation, when a WPB crosses the approximated cell boundary. The segmented WPBs were saved as ImageJ region of interest (ROI) sets for an entire plate of image stack as *_RoiSet.zip files (Data Citation 1) (Fig. 3a).

During feature extraction, both morphology and intensity-based WPB features were quantified (Table 1). Objects larger than $10 \mu \mathrm{m}^{2}$ were considered as virtually merged WPB clumps, that the microscope was unable to resolve separately. Therefore, those large objects were omitted using a size filter. The measured features with the well and FoV identifier of each WPB were saved in the comma separated *_Results_Features.csv ASCII files (Data Citation 1).

The WPB labelling was an essential step in order to assign each organelle to its influence zone. The mean pixel intensity of each WPB object was measured on the influence zone image. The number of rows (WPBs) in the resulted table was equal to the corresponding Results Feature files. The result of the measurements was saved in comma separated ASCII files named as *_Results_WBP_cell_ID.csv (Data Citation 1).

For QC purpose, the contours of the segmented and size filtered WPBs were overlaid in red on their original greyscale images and was saved as stack in RGB format in the * ${ }^{\star}$ WWF_overlay_RGB.zip (Data Citation 1) files. The overlay image facilitated visual inspection in order to identify vWF-rich areas, that were segmented as non-WPB artefacts (Fig. 3a).

\section{R script}

The morphological and intensity results of the WPBs were stored in ASCII tables and were evaluated statistically with Rscript.R (Data Citation 1) using $\mathrm{R}^{16}$, the well-established, open source statistical software (http://r-project.org). The $\mathrm{R}$ scripts calculated single values per well in each plate for:

(i) area ratio of WPBs with Feret's diameter larger than $1.5 \mu \mathrm{m}$ (1_PercentWPBarea),

(ii) WPB number per cell (2_TotalWPBnrPerCellNR),

(iii) WPB intensity per cell (3_TotalWPBrawIntDenPerCellNR, Fig. 1c) and

(iv) ratio of WPB number with Feret's diameter larger than $1.5 \mu \mathrm{m}$ (4_TotalFoVpercentWPBnr).

Representative phenotypes for hits versus controls are shown in Fig. 4.

In order to calculate the 1_PercentWPBarea feature for each well, first the total WPB was calculated (sum(FoVfeature\$Area)). Then the WPBs were selected with Feret's diameter larger than $1.5 \mu \mathrm{m}$ 

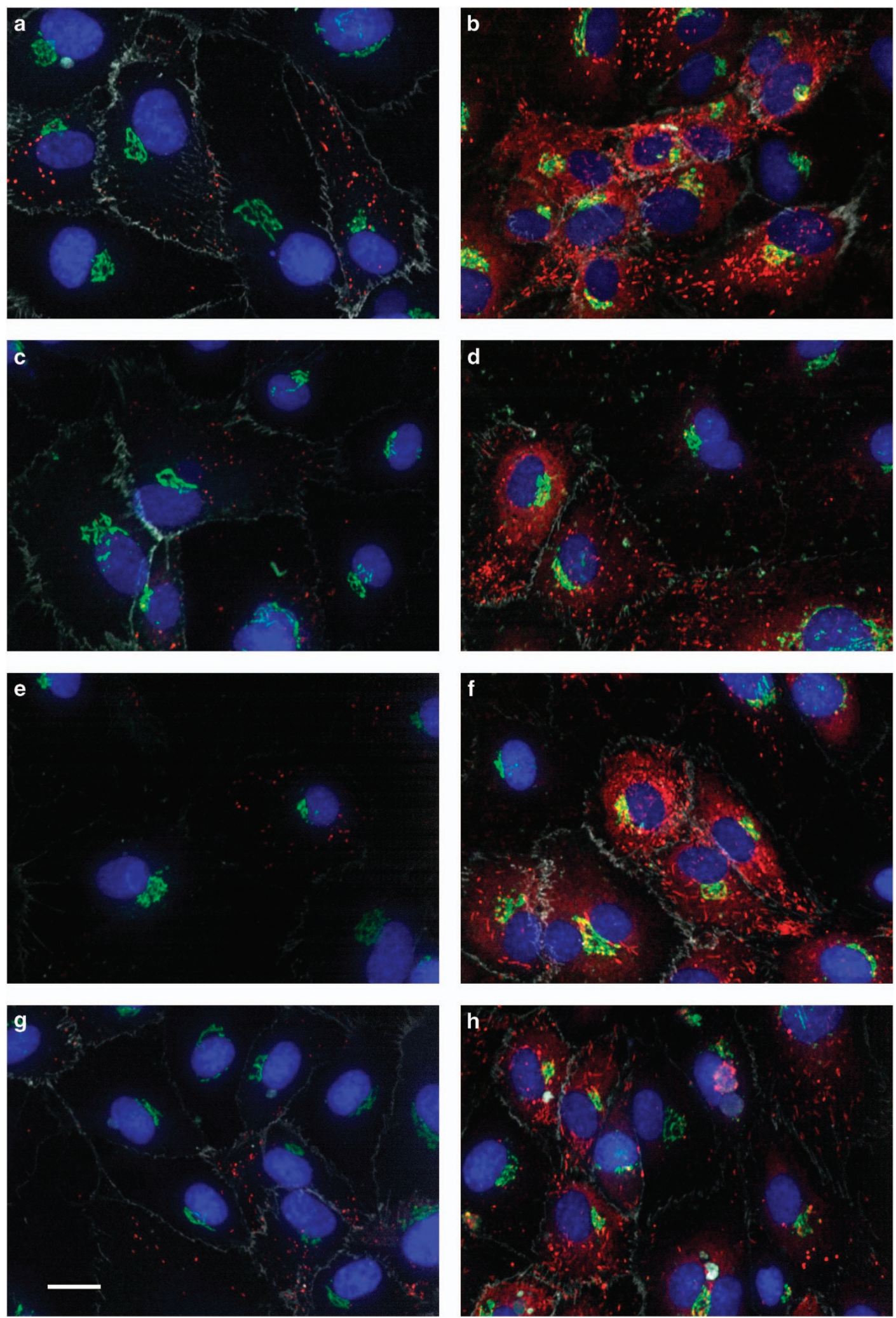

Figure 4. Representative control and hit phenotypes for all four biologically relevant parameters. siCTRL (left panels) and top hit phenotypes (right panels) for WPB area ratio control (a), top hit kinase DMPK (b), WPB number control (c), top hit kinase TRRAP (d), WPB intensity control (e), top hit kinase MAPK12 (f) and ratio of WPB with Feret diameter longer than $1.5 \mu \mathrm{m}$ control (g), top hit kinase MAP4K2 (h), respectively. Scalebar $20 \mu \mathrm{m}$. 
(over15umfeature), followed by calculating the total area of those WPBs (sum(over15umfeature $\$$ Area)) and their ratio (FoVpercentWBParea[f]). The mean of the 5 FoVs resulted the single PercentWPBarea value in each well.

The 2_TotalWPBnrPerCellNR feature was also calculated as a mean of five FoV ratios in each well. In each FoV the total number of WPBs (FoVTotalWPBnr) was devided by the total number of cells (FoVcellNR). The number of cellID values was extracted from the ${ }^{\star}$ _Results_WBP_cell_ID.csv file (Data Citation 1).

The WPB intensity per cell, 3_TotalWPBrawIntDenPerCellNR, was a ratio of the total intensity of all WPBs (sum(FoVfeature\$RawIntDen)) and the total number of cells (FoVcellNR). The mean of the five FoVs gave the single value per well.

The 4_TotalFoVpercentWPBnr was calculated as a ratio of the WPB number with Feret's diameter larger than $1.5 \mu \mathrm{m}$ (length(over15umfeature\$Area)) and the total number of WPBs (FoVTotalWPBnr) in a given FoV. The per-well value was calculated as the mean of the 5 FoV results.

The results of each plate were saved separately as ASCII files into subfolders named as 1_PercentWPBarea, 2_TotalWPBnrPerCellNR, 3_TotalWPBrawIntDenPerCellNR and 4_TotalFoVpercentWPBnr respectively.

The files have a 3 column data structure, that is required by the cellHTS2 package. The plate identifier in the first column (arbitrary string), the well identifier in the second column (e.g. A01, H12) and the measured/calculated single value in the third column.

\section{Bioconductor cellHTS2 script}

We used the cellHTS2 (ref. 17) package from Bioconductor in custom R scripts for statistical analysis. The workflow was prepared following the sample workflow described in 'End-to-end analysis of cell based screens: from raw intensity readings to the annotated hit list' (last retrieved on 09/02/2017 from https://www.bioconductor.org/packages/3.3/bioc/vignettes/cellHTS2/inst/doc/cellhts2Complete.pdf).

The file cellHTS2_VWF_1_PercentWPBarea.R contains an explanation of the method used for analysis. Initially, the working folder and the path to dataset was specified (these two folders can be identical). The dataset path was the folder where the Rscript.R result files were saved. The Platelist.txt, Plateconf.txt and Description.txt files (Data Citation 1) were prepared based on the sample workflow instruction. The dataset was normalised by the $\mathrm{B}$ score ${ }^{18}$ method. Following normalisation, the $\mathrm{Z}$ score (Fig. 3c) of each well was calculated and a report was generated in HTML format.

\section{Code availability}

The image analysis was performed under a 64 bit version of Kubuntu Linux 12.04, ImageJ version $1.50 \mathrm{f}$ and Fiji ${ }^{19}, \mathrm{R}$ version 3.2.5, cellHTS2 version 2.30.0.

The ImageJ_macro_vWF_HCS.ijm file is available at Harvard Dataverse repository (Data Citation 1).

The Rscript.R file is available at Harvard Dataverse repository.

All HTS statistical analysis R source code files (e.g. cellHTS2_VWF_1_PercentWPBarea.R) are available at Harvard Dataverse repository.

\section{Data Records}

The data associated with this work is available at the Harvard Dataverse repository (Data Citation 1).

All .zip files were renamed '.zip_' to circumvent the automatic extracting function of Harvard Dataverse repository. When downloading this data, the researcher needs to rename all files to *.zip again.

Image data files are named as A1.zip-K2.zip. After downloading, these files need to be unzipped into different folders.

The ImageJ_macro_vWF_HCS.ijm source code ASCII file contains the ImageJ/Fiji macro that perform the automated image analysis of a plate's image set.

Data resulting from the Delaunay Triangulation can be found in the * influzone.zip and in *_nuc-overlay_RGB.zip files. The *_vWF_overlay_RGB.zip files contain the segmentation contours of the WPBs overlaid on the original images and can be used for visual inspection QC. These contain images of a plate as stacks, do not require unzipping and can be opened directly with ImageJ or Fiji by the File/ Open menu.

*_Results_Nuc_Features.csv comma separated value ASCII files: the filename contains the plate number, the field 'Label' contains the well (row and column number) and FoV number, the field 'Mean' contains the influence zone identifier number of a given nucleus.

Segmented WPB contour data can be found for each plate in ${ }^{*}$ RoiSet.zip files. These files can be opened in ImageJ or Fiji using the Open function in ROI Manager.

The *_Results_Features.csv comma separated value ASCII files contain the measured WPB features.

The WPB labels, that connect them to a specific cell are stored in the ${ }^{\star}$ _Results_WBP_cell_ID.csv comma separated value ASCII files.

The Rscript.R source code ASCII file contains the statistical analysis of the measured WPB featured. It is a script written in $\mathrm{R}$ language.

The cellHTS2_VWF_1_PercentWPBarea.R, cellHTS2_VWF_2_TotalWPBnrPerCellNR.R, cellHTS2_VWF_3_TotalWPBrawIntDenPerCellNR.R and cellHTS2_VWF_4_TotalFoVpercentWBPnr. $\mathrm{R}$ ASCII files are R scripts. Those contain the source code of the HTS statistical analysis. 
The 1_PercentWPBarea_cellHTS2_SciData.zip,

2_TotalWPBnrPerCellNR_cellHTS2_SciData.zip,

3_TotalWPBrawIntDenPerCellNR_cellHTS2_SciData.zip and

4_TotalFoVpercentWBPnr_cellHTS2_SciData.zip files contain the input data, HTS analyses and HTML format reports, generated by their respective HTS statistical analysis R scripts. After downloading, these files need to be unzipped into different folders. The subfolder called as report-VWF* contains an index.html file that can be opened in any web browser.

\section{Technical Validation}

WPB segmentation: The ${ }^{*}$ _vWF_overlay_RGB.zip files contain the segmentation contours of the WPBs overlaid on the original images and can be used for visual inspection QC.

In order to assess the quality of the screen Z' factors were calculated to both replicates of all $4 \mathrm{WPB}$ HTS features, and available in the respective HTML reports.

Standard deviation was calculated across the replicates for all of the four WPB HTS features. It can be found in the HTML reports as reproducibility plate heatmaps (Fig. 3b).

\section{Usage Notes}

The user must rename the ${ }^{\star}$.zip_ files to ${ }^{\star}$.zip. The extension renaming was necessary in order to circumvent the automated decompress feature of Harvard Dataverse repository.

\section{References}

1. Ketteler, R., Kriston-Vizi, J. in Encyclopedia of Cell Biology Vol. 4, (eds Bradshaw R. A. \& Stahl P. D.) 234-244 (Academic Press, 2016).

2. Kiger, A. A. et al. A functional genomic analysis of cell morphology using RNA interference. J. Biol. 2, 27 (2003).

3. Boutros, M. et al. Genome-wide RNAi analysis of growth and viability in Drosophila cells. Science 303, 832-835 (2004).

4. Agrotis, A. \& Ketteler, R. A new age in functional genomics using CRISPR/Cas9 in arrayed library screening. Front Genet. 6, 300 (2015).

5. Buehler, E. et al. siRNA off-target effects in genome-wide screens identify signaling pathway members. Sci. Rep. 2, 428 (2012). 6. Blurton-Jones, M. et al. Neural stem cells improve cognition via BDNF in a transgenic model of Alzheimer disease. Proc Natl Acad. Sci. USA 106, 13594-13599 (2009).

7. Singh, S., Carpenter, A. E. \& Genovesio, A. Increasing the Content of High-Content Screening: An Overview. Journal of biomolecular screening 19, 640-650 (2014).

8. Ferraro, F. et al. A Two-Tier Golgi-Based Control of Organelle Size Underpins the Functional Plasticity of Endothelial Cells. Developmental Cell 29, 292-304 (2014).

9. Stevenson, N. L. et al. G protein-coupled receptor kinase 2 moderates recruitment of THP-1 cells to the endothelium by limiting histamine-invoked weibel-palade body exocytosis. Journal of Thrombosis and Haemostasis 12, 261-272 (2014).

10. Lopes da Silva, M. et al. Type II PI4-kinases control Weibel-Palade body biogenesis and von Willebrand factor structure in human endothelial cells. J. Cell. Sci. 129, 2096-2105 (2016).

11. Luft, C. \& Ketteler, R. Electroporation knows no boundaries: the use of electrostimulation for siRNA delivery in cells and tissues. $J$ Biomol Screen. 20, 932-942 (2015).

12. Zumbansen, M. et al. First siRNA library screening in hard-to-transfect HUVEC cells. J. RNAi Gene Silencing 6, 354-360 (2009).

13. Martin S. et al. in Assay Guidance Manual, (eds Sittampalam G. S. et al.) (2004).

14. Sternberg, S. R. Biomedical Image-Processing. IEEE Computer 16, 22-34 (1983).

15. Bernsen, J. in Proc. 8th Int. Conf. on Pattern Recognition (ICPR8) 1251-1255 (1986).

16. R Core Team: A Language and Environment for Statistical Computing (R Foundation for Statistical Computing: Vienna, Austria, 2016).

17. Boutros, M., Bras, L. P. \& Huber, W. Analysis of cell-based RNAi screens. Genome Biol. 7, R66 (2006).

18. Malo, N., Hanley, J. A., Cerquozzi, S., Pelletier, J. \& Nadon, R. Statistical practice in high-throughput screening data analysis. Nat. Biotechnol. 24, 167-175 (2006).

19. Schindelin, J. et al. Fiji: an open-source platform for biological-image analysis. Nat. Methods 9, 676-682 (2012).

\section{Data Citations}

1. Kriston-Vizi, J. Harvard Dataverse http://dx.doi.org/10.7910/DVN/GPXYGO (2016).

\section{Acknowledgements}

This work was supported by the UK Medical Research Council core funding to the MRC-UCL University Unit award code MC_U12266B, the Wellcome Trust (101472/Z/13/Z), and the European Union Seventh Framework Programme (FP7/2007-2013, grant PIRG08-GA-2010-276811).

\section{Author Contributions}

R.K., D.C., F.F. and J.K.V. designed and conceived the study. J.F., N.S. and F.F. performed experiments. J. K.V. performed all statistical and image analysis. R.K., J.K.V. and N.B. wrote the manuscript.

\section{Additional Information}

Supplementary information accompanies this paper at http://www.nature.com/sdata

Competing financial interests: The authors declare no competing financial interests.

How to cite this article: Ketteler, R. et al. Image-based siRNA screen to identify kinases regulating Weibel-Palade body size control using electroporation. Sci. Data 4:170022 doi: 10.1038/sdata.2017.22 (2017). 
Publisher's note: Springer Nature remains neutral with regard to jurisdictional claims in published maps and institutional affiliations.

(c) (i) This work is licensed under a Creative Commons Attribution 4.0 International License. The images or other third party material in this article are included in the article's Creative Commons license, unless indicated otherwise in the credit line; if the material is not included under the Creative Commons license, users will need to obtain permission from the license holder to reproduce the material. To view a copy of this license, visit http://creativecommons.org/licenses/by/4.0

Metadata associated with this Data Descriptor is available at http://www.nature.com/sdata/ and is released under the CCO waiver to maximize reuse.

(C) The Author(s) 2017 


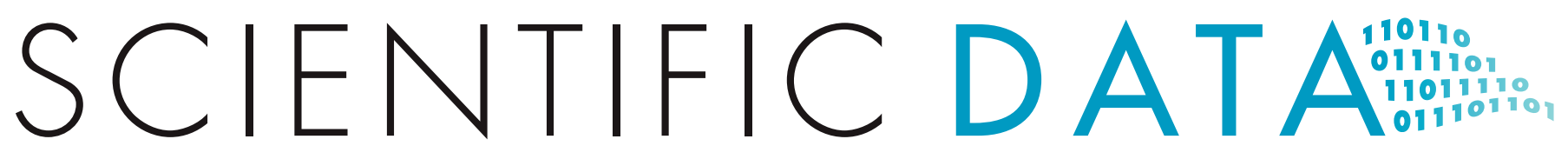

OPEN Corrigendum: Image-based siRNA

\title{
screen to identify kinases regulating Weibel-Palade body size control using electroporation
}

Robin Ketteler, Jamie Freeman, Nicola Stevenson, Francesco Ferraro, Nicole Bata, Dan F. Cutler \& Janos Kriston-Vizi

Scientific Data 4:170022 doi:10.1038/sdata.2017.22 (2017); Published 1 Mar 2017; Updated 27 Jun 2017

The authors regret that Nicola Stevenson was omitted in error from the author list of the original version of this Data Descriptor. This omission has now been corrected in the HTML and PDF versions of this Data Descriptor, as well as the accompanying Corrigendum.

Contributions

R.K., D.C., F.F. and J.K.V. designed and conceived the study. J.F., N.S. and F.F. performed experiments. J. K.V. performed all statistical and image analysis. R.K., J.K.V. and N.B. wrote the manuscript.

\begin{abstract}
(c) (i)
Open Access This article is licensed under a Creative Commons Attribution 4.0 International License, which permits use, sharing, adaptation, distribution and reproduction in any medium or format, as long as you give appropriate credit to the original author(s) and the source, provide a link to the Creative Commons license, and indicate if changes were made. The images or other third party material in this article are included in the article's Creative Commons license, unless indicated otherwise in a credit line to the material. If material is not included in the article's Creative Commons license and your intended use is not permitted by statutory regulation or exceeds the permitted use, you will need to obtain permission directly from the copyright holder. To view a copy of this license, visit http://creativecommons. org/licenses/by/4.0/
\end{abstract}

(C) The Author(s) 2017 